\section{Effects of an} educational intervention on the anxiety of women awaiting mastectomies

By France Provençal Belleau, Louise Hagan, and Benoît Mâsse

\section{Abstract}

The purpose of this experimental study was to assess the effects of an individualized psychocognitive educational intervention on preoperative anxiety in women awaiting mastectomies. A total of 60 women aged between 27 and 65 years were randomly distributed to two groups of 30 participants. Using the Situational Anxiety Inventory (IAS) along with repeated-measures variance analysis, it was noted that, immediately following the educational interventions (both experimental and control) there was a significant reduction $(p<0.01)$ of anxiety with the experimental group having an additional reduction of 4.83 ( $p=0.05)$. The day before surgery, only the experimental educational intervention resulted in a significant reduction $(p=0.03)$ of anxiety, but the difference between the two educational interventions was not significant. These results appear to support Lazarus and Folkman's stress, appraisal and coping theory which states that direct action on cognitive perception can influence a feeling of threat as assessed through the level of anxiety.

The National Cancer Institute of Canada (NCIC, 2000) estimates that one in 9.5 women will develop breast cancer during her lifetime. Canadian breast cancer rates are said to be among the highest in the

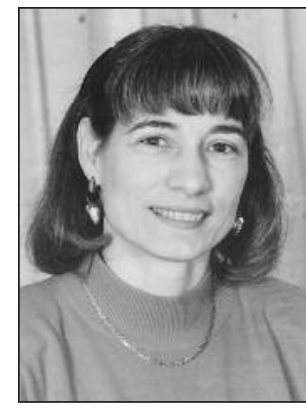

France Provençal Belleau

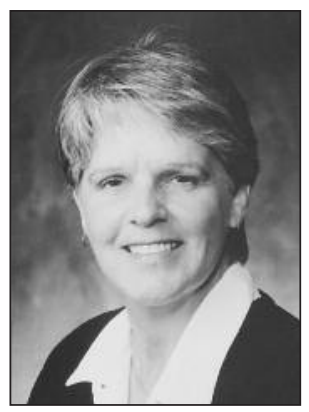

Louise Hagan

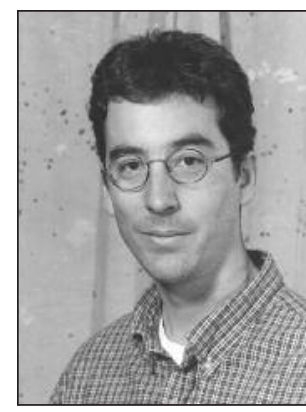

Benoît Mâsse world (Gaudette, Silberberger, \& Atwell, 1994). During the 19751992 period, the crude mortality rate related to breast cancer in women increased by 69.6\% in Quebec (Beaupré, 1995). Breast cancer ranks first among the cancers affecting women in this Canadian province (Beaupré; NCIC).

When cancer is diagnosed, women must have access to quality hospital medical and nursing services, but in Quebec, this is taking place in a context where the shift to ambulatory care is proceeding very fast, thus disturbing professional practices and relationships with patients (MSSS, 1996). One of the impacts of this organizational change has been a reduction of the time women requiring surgery are exposed to nurses' professional interventions, especially the educational intervention aimed at preparing them for this surgery in the best possible way.

\section{The problem}

A great deal of anxiety usually occurs with the discovery of cancer and the wait for a mastectomy (Northouse, 1989; Wainstock, 1991). This anxiety is the result of fears related to various factors: the surgery itself, apprehension of pain, mutilation, effects on body image, extent of cancer, death, loss of sexual attractiveness, and loss of control (Ali \& Khalil, 1991; Lierman, 1984; Northouse; Reaby, Hort, \& Vandervord, 1994; Royak-Schaler, 1992; Wainstock). In varying degrees, this anxiety affects the women's ability to receive and retain the information provided through pre-operative teaching (Haines, 1992; Lamarche, 1993; Oberle, Allen, \& Lynkowski, 1994; Ruzicki, 1989; Young, De Guzman, Matis, \& McClure, 1994).

The provision of adequate information and emotional support is an avenue that is likely to increase the sense of control required to reduce anxiety and consequently to cope with the stress associated with the mastectomy (Palsson \& Norberg, 1995).

Most often the content taught to women is standardized. Thus it is not necessarily particular and directly related to the perceived needs of the women themselves at this episode of care. The content often deals with technical aspects (physical preparation for surgery, expected physical effects, respiratory exercises, and motor skills to be acquired). The expression of emotions and active listening of fears or concerns are not part of the systematic priorities of the educational interventions even when we know that such an approach can potentially have a positive impact in reducing anxiety (Hathaway, 1986; Richardson \& O'Sullivan, 1991).

The purpose of this experimental study was to assess the effects of an individualized, psychocognitive, educational intervention on preoperative anxiety in women awaiting mastectomies.

\section{Theoretical framework}

This study is based on Lazarus and Folkman's (1984) stress coping theory. This theory explains how an individual facing a stressful situation performs a cognitive assessment (primary, secondary, or reassessment) and deals with the problem using various coping strategies. As applied to the context of this study, Lazarus and Folkman's theory helps identify the factors perceived by women as threatening their physical, psychological, and social integrity and helps prioritize the educational interventions aimed at these stress factors. The educational interventions are meant to be an external resource and, thus, provide coping assistance to women by providing answers to their search for information. These interventions can change the cognitive assessment of events perceived as being stressful and, consequently, reduce the associated situational anxiety.

France Provençal Belleau, RN, MSc, is Head, Breast Cancer Screening Program, Quebec City Region, Hôpital du Saint-Sacrement du Centre Hospitalier Affilié de l'Université Laval de Québec (CHAUQ). Louise Hagan, RN, PhD is a professor, Faculté des sciences infirmières, Université Laval, Québec City, QC. Benoît Mâsse, PhD, is a biostatistician at the Centre de Recherche, CHAUQ. 


\section{The research hypothesis}

That immediately after the educational intervention and on the day before surgery there will be a greater reduction in the anxiety level in women who participated in an individualized, psychocognitive, educational intervention than in women who only received the standard educational intervention.

\section{Methodology}

\section{Study design}

This is a pre-test/post-test experimental study.

\section{Population and sample}

The population in the study was composed of women from eastern Quebec who had been diagnosed with breast cancer and were scheduled to have surgery (simple mastectomy or segmentectomy with or without node dissection or modified radical mastectomy).

They had to be between the ages of 21 and 65 , and able to read and speak French. Exclusion criteria were the taking of anxiolytic medication on a regular basis or a psychiatric follow-up because of the bias that these factors could have on the measurement of situational anxiety among these patients.

The women were recruited in an acute care hospital in the Quebec City area between September 28 and December 18, 1998. Sixty-four women were selected in total and 60 of them agreed to take part in the study. Three women refused due to lack of time, fatigue, or lack of interest in completing forms, and one woman dropped out during the study. Women were then randomly assigned to the experimental group (E) or control group (C) with the help of a table using alphabetical letters. In both groups, the participants could be accompanied by a significant other or a family member if so desired.

\section{Study variables}

\section{Independent variable}

The individualized, psychocognitive, educational approach was the study's independent variable. It was defined as a combination of both psychotherapeutic and cognitive approaches. The psychotherapeutic dimension of the experimental educational intervention involved exploring and systematically supporting each woman in the expression of her feelings and emotions about the surgery, cancer diagnosis, spousal relationship, or any other aspects that represented a concern or a source of anxiety. It also allowed for the exploration of the coping strategies used by the woman and encouraged information-seeking. At the meeting, each woman was given the necessary time to fully express what she was feeling at that time and to ask all the questions she wanted. When needed, the participant was offered a few minutes of relaxation (e.g. controlled diaphragmatic breathing) to alleviate anxiety. When emotions were expressed (e.g. crying) touching could represent a form of support. The cognitive dimension of the educational intervention was first meant to respond to the specific informational needs expressed by the participants. A minimum level of learning was thus ensured, i.e. the acquisition and understanding of information provided in response to the perceived needs of the woman herself and the nurse.

Teaching tools used were anatomical charts, demonstration material, an information brochure about the surgery, and a video on the stay in the operating area.

This experimental intervention was compared to the regular educational intervention which was oriented towards a more direct cognitive approach and based mainly on more standardized education covering information related to the pre-, peri-, and post-operative mastectomy periods. The regular educational intervention could also occasionally involve active listening and psychological support, but it did not systematically prioritize the women's expressions of perceived threats and their associated feelings and emotions.

\section{Dependent variable}

The main dependent variable in the study was the level of situational anxiety.

\section{Measurement instruments and data collection}

The data were collected by means of self-administered questionnaires completed at three different times: pre-educational intervention (about 14-19 days before the surgery), post-educational intervention (immediately after the educational intervention, about 14-19 days before surgery), and presurgery (the day before surgery). The first questionnaire was completed upon joining the study. It included sociodemographic variables, variables related to the history of the present illness, the existence of a confidante, and the wait times (time between diagnosis and the educational intervention and between the educational intervention and surgery), as well as measurement of pre-operative situational anxiety. The second and third questionnaires again measured the anxiety level.

The Situational Anxiety Inventory (IAS) was the instrument used to measure the pre-operative anxiety level in women awaiting mastectomies before and after the educational intervention, as well as on the day before surgery. The IAS is a French-Canadian adaptation of one of two questionnaires in the revised version of the State-Trait Anxiety Inventory - Form Y (STAI-Y) by Spielberger (1983) as translated by Gauthier and Bouchard (1993).

The IAS takes the form of a self-administered questionnaire composed of 20 sentences that assess the subject's current emotional state. The respondent is required to indicate the intensity of his/her feelings on a four-point Likert scale ranging from 1, "Not at all" to 4, "A lot". There is an equal distribution of statements (10 each) indicating the presence or absence of unpleasant emotional states (Bouchard, 1990). Ratings can vary between 20 and 80. Lower ratings reflect calm and relaxation, mid-range ratings correspond to moderate levels of stress and apprehension, while higher ratings indicate significant fear, apprehension, and panic (Bouchard). This study's results with 60 subjects reveal good homogeneity among the items (Cronbach's Alpha=0.95).

\section{Results}

\section{Respondents' profile}

There were no statistical differences in the sociodemographic characteristics between the experimental group (individualized psychocognitive intervention) and the control group (standard intervention). In the control group, the age of the 30 women varied between 36 and 64 (M 51.50; S.D. 7.61) while that of their counterparts in the experimental group varied from 27 to 65 (M 52.17; S.D. 9.00). There was, however, a significant difference in the length of the educational intervention: the educational intervention for the experimental group (M 78.50 min.; S.D. 21.38) was longer $(\mathrm{p}<0.01)$ than that of the control group (M 50.93 min.; S.D. 16.97). A significant difference $\left(\mathrm{X}^{2}=4.80 ; \mathrm{df}=1 ; \mathrm{p}=0.03\right)$ was noted with regard to the frequency of psychosocial referrals (a social worker working with the referred women) as there were many more referrals for women in the experimental group $(46.7 \%)$ than in the control group (20\%).

\section{Pre-operative anxiety}

The pre-operative anxiety level was measured in both groups before the educational intervention, immediately after, and the day before surgery (see Table One). No significant difference was present between the two groups before the educational intervention $(\mathrm{t}=1.57$; $\mathrm{df}=58 ; \mathrm{p}=0.12$ ).

In order to verify the effectiveness of the corresponding educational intervention, a repeated-measurement analysis of variance was done by means of the PROC MIXED procedure using SAS 6.12. Two measured variables are presented in Table Two, the first representing the effect immediately after the educational 
intervention and the other, the effect just before surgery (on the day before). A positive value indicates reduced anxiety. One observes that immediately after the educational intervention both types of educational interventions resulted in significant anxiety reduction, with the experimental group's educational intervention showing a significant additional reduction of anxiety of 4.83 when compared to the control group's educational intervention (10.53 versus 5.70).

Moreover, only the experimental group's educational intervention resulted in significant anxiety reduction until the day before surgery. Otherwise, there was no significant difference between the two groups regarding the educational intervention's effectiveness the day before surgery.

The results of the Spearman rank correlation tests and variance analysis showed that none of the sociodemographic characteristics associated with the history of the present disease, the existence of a confidante, nor contextual characteristics were connected to the anxiety level before the educational intervention.

We checked to see if the effectiveness of the educational interventions (for both groups) was linked to the respondents' characteristics. Variance analysis allowed us to verify the homogeneity of the effectiveness of the educational intervention among the various categories of marital status (i.e. single, married/living common-law, separated/widowed) and education level.

In the experimental group we observed that when a woman was accompanied to the educational intervention, there seemed to be a link between that fact and the effectiveness of the educational intervention immediately after the educational intervention $\left(r^{2}=0.43 ; p=0.02\right)$ and on the day before surgery $\left(\mathrm{r}^{2}=0.43 ; \mathrm{p}=0.02\right)$. These positive correlations, while weak, mean that the educational intervention seems to be more effective when the woman is accompanied. We also noticed a negative correlation $\left(r^{2}=-0.41 ; p=0.03\right)$ between having a family history of breast cancer and the effectiveness of the educational intervention. A significant response $(\mathrm{p}=0.02)$ was observed showing that a difference in the effectiveness of the interventions was related to differences in marital status. Indeed, married women or women in common-law relationships had a lower anxiety level immediately after the experimental educational intervention.

Table One: Pre-operative anxiety levels at the three measurement points

\begin{tabular}{|l|cc|cc|}
\hline & \multicolumn{2}{|c|}{$\begin{array}{l}\text { E Group }(\mathrm{n}=30) \\
\text { Individualized intervention }\end{array}$} & $\begin{array}{l}\text { C Group }(\mathrm{n}=30) \\
\text { Standard intervention }\end{array}$ \\
\hline & Average $\quad$ St. deviation & Average St. deviation \\
\hline $\begin{array}{c}\text { Anxiety before the educational } \\
\text { intervention: IAS \#1 }\end{array}$ & 52.00 & 15.32 & 46.33 & 12.58 \\
\hline $\begin{array}{c}\text { Anxiety immediately after the } \\
\text { educational intervention: IAS \#2 }\end{array}$ & 41.47 & 12.18 & 40.63 & 11.98 \\
\hline $\begin{array}{l}\text { Anxiety the day before surgery: } \\
\text { IAS \#3 }\end{array}$ & 47.03 & 14.45 & 44.90 & 11.76 \\
\hline
\end{tabular}

Table Two: Effectiveness of the educational intervention on anxiety reduction immediately after the educational intervention and the day before surgery
Effectiveness

Effect immediately after the

educational intervention: IAS \#1 - IAS \#2

Effect the day before surgery:

IAS \#1 - IAS \#3
E Group $(\mathrm{n}=30)$

Reduction in anxiety $\mathrm{p}$ value
Individualized intervention
In the control group, previous surgery for breast cancer seemed to be linked $\left(r^{2}=-0.38 ; p=0.04\right)$ to the effectiveness of the educational intervention on the day before surgery, as was the waiting period (educational intervention to surgery) $\left(\mathrm{r}^{2}=-0.40 ; \mathrm{p}=0.03\right)$. It appeared that the longer the wait between educational intervention and surgery, the lower the effectiveness of the educational intervention on the day before surgery. However, the number of subjects $(n=5)$ who had previous breast cancer surgery was too low to establish a conclusive relationship. A study with a larger sample could possibly confirm this relationship.

Also, in both the experimental $\left(\mathrm{r}^{2}=0.41 ; \mathrm{p}=0.03\right)$ and control $\left(\mathrm{r}^{2}=0.42 ; \mathrm{p}=0.02\right)$ groups, an association was observed between the anxiety level on the day before surgery and a referral to a psychosocial resource (social worker) at the end of the educational intervention. Indeed, women $(n=20)$ who had been referred to a psychosocial resource had, on the day before surgery, a reduction of their anxiety level of 11.08 (S.D. 13.03) in the experimental group $(n=14)$ and of 11.00 (S.D. 6.16) in the control group $(n=6)$ when compared to their anxiety level before the educational intervention. The women who expressed the desire to meet with a psychosocial resource were referred after the educational intervention (this service was offered to all the women awaiting mastectomies). It was up to them whether they contacted the social worker before or after surgery. However, no analyses were done regarding pre-operative contact (whether seen or not), contact type (telephone, interview, etc.), and the number of interventions, but rather whether or not there was a psychosocial referral made (referred/non-referred).

\section{Discussion}

The additional intervention by the psychosocial resource (the social workers to whom some of the women were referred) before surgery potentially introduced a bias in the results for the third preoperative anxiety measurement, i.e. on the day before surgery. This variable (psychosocial referral) should have been controlled by doing specific analysis (contacted before surgery/not contacted) in order to assess its impact on the third measurement. Furthermore, as this service was offered by two social workers, we would have had to ensure the consistency of the psychosocial intervention. Thus, this represents a limitation to this study.

The general population's level of anxiety assessed by the State-Trait Anxiety Inventory (STAI) - Spielberger (1983) is established with categories for age and gender. For women between 50 and 54 years old, the average anxiety level is 32.60 (S.D. 7.30). One standard deviation higher than average indicates a high anxiety level (39.90). This means that some of the women who participated in this study (with an average age of 52) had a high level of anxiety before the educational intervention since their level of anxiety was greater than
C Group $(\mathrm{n}=30)$

Standard intervention

Reduction in anxiety $\mathrm{p}$ value
Difference between the two groups Reduction in anxiety $\mathrm{p}$ value

$$
10.53
$$$$
<0.01
$$

4.84

0.03
$<0.01$

0.51

1.43
4.83

3.41

0.27 
39.90 , the standard deviation being higher than the normative average with averages of 46.33 (S.D. 12.58) and 52.00 (S.D. 15.32) in the control and experimental groups, respectively. Thus, the perception of threats linked to breast cancer and surgery appears to be real.

The individualized psychocognitive educational intervention was intended to reduce the level of anxiety by directly influencing the perception of threat and by satisfying the need for information related to the distressing event. The study results show that immediately after the educational intervention, it was observed that the two types of educational interventions, experimental and control, significantly reduced the anxiety level.

While few studies have assessed the impact of an individual educational intervention done by a nurse with clients awaiting surgery, the results of this study appear to be related to those of Shimko's study (1981) with 81 clients awaiting neurosurgery where it was observed that the anxiety level dropped by an average score of 6.0 immediately after an individual or small-group educational intervention focusing on cognitive and psychomotor aspects. The results suggest that giving only information is enough to reduce the anxiety level immediately after the educational intervention.

The study by Richardson and O'Sullivan (1991) using a quasiexperimental design with 60 clients awaiting elective surgery showed that the group that received an individual educational intervention on the day prior to surgery had an anxiety level significantly lower (F $[1.58]=4.97 ; \mathrm{p}<0.05)(\mathrm{M} 40.43$; S.D. 9.76) than the group that did not receive any such intervention (M 47.16; S.D. 14.97). The educational intervention provided support to the client. The content focused on fears or apprehensions and issues related to the surgery.

Clinically, with the IAS, a reduction of 5.7 in the anxiety level means that two statements out of 20 (i.e. $10 \%$ of the statements) have been reduced by two and that another one has dropped by one, or that five statements (i.e. $25 \%$ of the statements) have been reduced by one, which could be considered significant. A reduction of 10.53 in the level of anxiety represents five statements out of 20 (i.e. $25 \%$ of the statements) that have incurred a drop of two on the Likert scale or 10 statements (i.e. $50 \%$ of the statements) that have dropped by one, for instance, which represents an additional significant reduction. An added reduction of the anxiety level equal to 4.83 is therefore significant for women awaiting mastectomies.

In the control group, the educational intervention started with the pre-admission health record (checking on completion of diagnostic tests and pre-operative consultations and usual data collection). Fears or concerns regarding the surgery, hospital stay, or return home were examined, but we did not explore the fears, real or anticipated, resulting from the cancer diagnosis and mastectomy announcement, nor the relationship with the spouse and family since the diagnosis. The coping strategies used by the women to problem-solve, and their existing support network represented other elements that were explored with the women but that did not play a leading role. However, the teaching material used and the informational content aimed at preparing women for surgery and self-care were the same for both types of educational intervention and these similarities probably contributed to reducing preoperative anxiety. This could also explain why the difference between these two educational interventions is not larger than 4.83 .

It is possible that the length of the educational intervention administered to the experimental group, which is significantly greater than that of the control group $(\mathrm{p}<0.01)$, also contributed in a noticeable way to the reduction of the anxiety level in this group. The greater length of time devoted to active listening and expression of emotions may potentially contribute to further reducing anxiety by helping women communicate all of their concerns and questions related to the surgery and breast cancer diagnosis and to receive needed answers.

With a high level of anxiety, which is the case for the women in this study, Lazarus and Folkman (1984) state that the individual will use emotion-focused strategies before using problem-focused strategies. The fact that the experimental educational intervention was focused on the expression of feelings and emotions and on support may have resulted in a more pronounced impact on anxiety reduction as the approach used was more closely related to an emotion-focused coping strategy. We are inclined to believe that this sizeable reduction of anxiety in the experimental group was in a large part due to the very nature of the experimental intervention.

The context of the experimental educational intervention, for instance the fact that the meetings took place in a different room (friendly and intimate) than that used by the control group, may have also fostered better feelings in the women and helped lower their anxiety levels.

In the experimental group, some of the effects of the educational intervention on anxiety reduction seem to last until the day before surgery ( 4.84 reduction; $p=0.03$ ). However, the difference between the effect of both types of educational interventions on the day before surgery is not significant $(\mathrm{p}=0.27)$. Thus, one cannot claim an increased effectiveness of the experimental intervention.

In the experimental group, the effectiveness of the educational intervention appears to be associated with attending with a companion $\left(\mathrm{r}^{2}=0.43 ; \mathrm{p}=0.02\right)$. The individualized psychocognitive approach allowed the women to be accompanied. During the educational intervention, the significant other's (friend, spouse, or family member) participation and support were sought and encouraged. This may have contributed to a further decrease in the anxiety level. Moreover, women were invited to attend the education intervention with a companion in both the experimental and control groups. However, in the control group's educational intervention, there was no systematic strategy for inviting participation and support from the women's companions.

\section{Conclusion}

The nurse is in a privileged position to help and support women awaiting mastectomies. It would appear that an educational intervention during the pre-operative period can help reduce anxiety in these women. A psychocognitive-based educational intervention also appears to help further reduce their anxiety.

The results of this study confirm the importance of planning and development of educational interventions for this population. This study especially demonstrated the importance of focusing educational interventions not only on surgery-related cognitive aspects but also on the psychological aspects (expression of fears, threats, apprehensions) linked both to the surgery and in particular to the cancer diagnosis. It is increasingly vital that efforts be sustained and encouraged to improve the quality of the education provided to women awaiting mastectomies in spite of environments that can sometimes not be conducive to the humanization and individualization of nursing interventions. It may also be useful to consider some form of follow-up for these women between the time of the educational intervention and the day before surgery in order to maintain an acceptable anxiety level for optimal quality of life during this difficult episode of their breast cancer treatment. Such follow-up, via telephone or in any other form, could be the topic of further research and could help determine if clinical practices need to be changed so that women can benefit from an educational intervention aimed at reducing their anxiety until the day before surgery.

Additional studies could also help identify the specificity of the educational approaches used in pre-operative education in order to better assess the contribution of the various elements of the assessed intervention. It would also be necessary to better control the role of some confounding variables such as referrals to other psychosocial resources, especially when one wants to measure the duration of the reduction in anxiety levels until the day before surgery. Moreover, one should ensure that the physical environment for the educational intervention be identical for the groups under comparison. 


\section{References}

Ali, N.S., \& Khalil, H.Z. (1991). Identification of stressors, level of stress, coping strategies and coping effectiveness among Egyptian mastectomy patients. Cancer Nursing, 14(5), 232-239.

Beaupré, M. (1995). Rapport annuel des nouveaux cas de cancer déclarés au fichier des tumeurs du Québec, année 1992. Montréal, PQ: Ministère de la Santé et des Services sociaux, Gouvernement du Québec.

Bouchard, S. (1990). Traduction, fidélité et validité d'un inventaire d'anxiété situationnelle et de trait d'anxiété. Mémoire de maîtrise inédit, Université Laval, Québec.

Gaudette, L., Silberberger, C., \& Atwell, K. (1994). Comprendre la statistique du cancer du sein. La filière du cancer. Bulletin des registres de cancer au Canada, 11, 4-5.

Gauthier, J., \& Bouchard, S. (1993). Adaptation canadienne française de la forme revisée du State-Trait Anxiety Inventory de Spielberger. Revue Canadienne des Sciences et du Comportement, 23, 559-578.

Haines, N. (1992). Same day surgery: Coordinating the education process. Association of Operating Room Nurses Journal, 55(2), 573-580

Hathaway, D. (1986). Effect of preoperative instruction on postoperative outcomes: A meta-analysis. Nursing Research, 35(5), 269-275.

Institut National du Cancer du Canada. (2000). Statistiques canadiennes sur le cancer, 2000. Toronto, Canada: Author.

Lamarche, D. (1993). Enseignement préopératoire structuré. L'infirmière canadienne, 89(4), 38-41.

Lazarus, R.S., \& Folkman, S. (1984). Stress, appraisal and coping. New York: Springer Publishing Company.

Lierman, L.M. (1984). Support for mastectomy: A clinical nursing research study. Association of Operating Room Nurses Journal, 39(7), 1150-1157.

Ministère de la Santé et des Services sociaux. (1996). La santé et les services sociaux - Enjeux et orientations stratégiques d'un système en transformation. Montréal, PQ: Gouvernement du Québec.
Northouse, L.L. (1989). The impact of breast cancer on patients and husbands. Cancer Nursing, 12(5), 276-284.

Oberle, K., Allen, M., \& Lynkowski, P. (1994). Follow-up of same day surgery patients: A study of patient concerns. Association of Operating Room Nurses Journal, 59(5), 1016-1025.

Palsson, M.-J.E., \& Norberg, A. (1995). Breast cancer patients' experiences of nursing care with the focus on emotional support: The implementation of a nursing intervention. Journal of Advanced Nursing, 21(2), 277-285.

Reaby, L.L., Hort, L.D., \& Vandervord, J. (1994). Body image, selfconcept, and self-esteem in women who had a mastectomy and either wore an external breast prosthesis or had breast reconstruction and women who had not experienced mastectomy. Health Care for Women International, 15, 361-375.

Richardson, M., \& O'Sullivan, S. (1991). Preoperations interviews: A nursing intervention to reduce patients' anxiety. The Australian Journal of Advanced Nursing, 8(3), 3-5.

Royak-Schaler, R. (1992). Psychological processes in breast cancer: A review of select research. Journal of Psychosocial Oncology, 9(4), 71-89.

Ruzicki, D.A. (1989). Realistically meeting the educational needs of hospitalized acute and short-stay patients. Nursing Clinics of North America, 24(3), 629-637.

Shimko, C. (1981). The effect of preoperative instruction on state anxiety. Journal of Neurosurgical Nursing, 13(6), 318-322.

Spielberger, C.D. (1983). Manual for the State-Trait Anxiety Inventory (Form Y). Palo Alto, CA: Consulting Psychologist Press.

Wainstock, J.M. (1991). Breast cancer: Psychosocial consequences for the patient. Seminars in Oncology Nursing, 7(3), 207-214.

Young, R., De Guzman, C.P., Matis, M.S., \& McClure, K. (1994). Effect of preadmission brochures on surgical patients' behavioral outcomes. Association of Operating Room Nurses Journal, 60(2), 232-241 